\title{
ACUPUNTURA, VENTOSA TERAPIA E TERMOGRAFIA NO TRATAMENTO DE LESÃO MUSCULAR: RELATO DE CASO
}

\section{ARTIGO ORIGINAL}

VALLE, Ana ${ }^{1}$

VALLE, Ana. Acupuntura, ventosa terapia e termografia no tratamento de lesão muscular: relato de caso. Revista Científica Multidisciplinar Núcleo do Conhecimento. Ano. 06, Ed. 10, Vol. 06, pp. 132-143. Outubro 2021. ISSN: 24480959, Link de acesso: https://www.nucleodoconhecimento.com.br/saude/ventosaterapia, DOI: 10.32749/nucleodoconhecimento.com.br/saude/ventosa-terapia

\section{RESUMO}

A Medicina Alternativa tem sido utilizada como tratamento natural pelos atletas com o intuito de evitar a utilização de medicação alopata, promovendo, assim, um equilíbrio físico e mental. Atualmente, a Medicina Tradicional Chinesa, tem despertado o interesse científico a fim de comprovar sua eficácia como Medicina Alternativa, sendo a Acupuntura um meio de tratamento que faz parte desta. A Ventosa Terapia, também, vem se destacando como alternativa para diversos profissionais da área de saúde como tratamento para dores musculares. A termografia, muito utilizada pelos fisioterapeutas para diagnósticos e uma visualização pontual das lesões musculares, é apresentada em conjunto com a acupuntura e ventosa terapia, nesse estudo de caso, como recurso complementar para o tratamento de lesão muscular. Tendo em vista este contexto, tem-se como questão norteadora: seria possível optar pela Medicina Alternativa, associando recursos modernos como a acupuntura, ventosa terapia e termografia para tratamento pontual das lesões musculares? Tendo como intuito apresentar um relato de caso em que o objetivo é demonstrar que um recurso atual como a termografia pode complementar uma técnica milenar como a Acupuntura e a

\footnotetext{
${ }^{1}$ Pós-graduação em Didática do Ensino Superior, Especialista em Acupuntura e Eletroacupuntura, Tecnólogo em Radiologia.
}

RC: 99476

Disponível em: https://www.nucleodoconhecimento.com.br/saude/ventosa-terapia 
ventosa terapia. Usando o resultado dos exames termográficos com as principais queixas do paciente, foi realizada uma anamnese e proposto um tratamento preciso na Medicina Tradicional Chinesa, visando a realização de um tratamento mais natural sem a utilização de medicamentos alopáticos com eficácia de 100\% no tratamento.

Palavras-chaves: Acupuntura, Lesão, Termografia, Ventosa.

\section{INTRODUÇÃO}

A Acupuntura é uma prática milenar que faz parte da chamada Medicina Tradicional Chinesa. Tem sido praticada por milhares de anos na China e alguns países do continente asiático. É descrita como efetiva no tratamento de diversas doenças e condições, sendo considerada um instrumento útil na prática da saúde. Da prática primitiva até os dias de hoje, a acupuntura, tem ganhado novos adeptos, atraindo, assim, a atenção da comunidade científica, a fim de entender melhor seu mecanismo de ação e sua efetividade. Estudos têm sido feitos e publicado envolvendo acupuntura, entretanto, ainda há controvérsia acerca de sua eficácia e efetividade. (LIN, 2013)

A Medicina Ocidental define a acupuntura como sendo a estimulação de pontos específicos localizados na superfície da pele, que tem a capacidade de alterar diferentes condições fisiológicas e bioquímicas no corpo, a fim de se obter o efeito desejado. Os pontos de acupuntura são áreas de sensibilidade, nas quais são introduzidas agulhas para estimular diversos receptores sensoriais que, por sua vez, estimulam os nervos que enviam impulsos para o hipotálamo e para a hipófise, localizados na base do cérebro. Está comprovado que o estímulo pela acupuntura pode liberar endorfinas, neurotransmissores e neuro-hormônios, melhorando o fluxo sanguíneo e estimulando o sistema imunológico. (SIGNIFICADOS, 2011)

A terapêutica com aplicação de ventosa ou, simplesmente, ventosa terapia, é uma antiga prática tradicional da Medicina Chinesa, onde uma estrutura específica é posicionada sobre a pele do paciente e produz efeitos terapêuticos através da 
criação de um vácuo no interior desta estrutura ou instrumento de modo que a pele e tecidos superficiais sejam sugados e mantidos no interior do corpo. (FILHO, 2016)

A termografia é um exame muito utilizados pelos fisioterapeutas, sendo utilizada como método de diagnóstico capaz de apontar alterações metabólicas e fisiológicas que podem revelar a extensão de lesões e suas áreas dolorosas, confirmando ou alterando diagnósticos clínicos, possibilitando a visualização das alterações térmicas da superfície cutânea. A imagem térmica digitalizada de alta resolução gerada é conhecida como termograma e pode-se proceder avaliação quantitativa (baseada em critérios térmicos) e qualitativa (baseada em critérios não térmicos, como por exemplo; área, distribuição, forma e simetria) das imagens. Este é um exame de Diagnóstico Funcional, que é dado pelo padrão de assimetria, e reflete a fisiologia ou a fisiopatologia da $\mathrm{ROI}$ ou da região à distância da ROI. Os achados deste devem ser correlacionados com as queixas do paciente e manifestações clínicas. (REVISTA SOCIEDADE BRASILEIRA DE FISIOTERAPIA, 2014)

A termografia é um método não invasivo e sem contato utilizado para registrar padrões térmicos corporais, utilizada para captar o calor emitido pelo corpo ou partes deste, portanto, pode ser utilizado como diagnóstico de lesões causadas pelo treinamento.

A análise de imagens infravermelhas como diagnóstico tem como vantagens: baixo custo; é uma técnica não invasiva; indolor; sem contato; não-intrusiva; sem radiação ionizante; inofensiva; disponibiliza as temperaturas de uma superfície em imagens de tempo real; possibilita a localização da lesão e é capaz de demonstrar mudanças metabólicas e fisiológicas através de um exame funcional, e não é restrita aos detalhes anatômicos como em outros métodos de análise. (BANDEIRA et al., 2014)

Portanto, o presente relato de caso tem como questão norteadora: seria possível optar pela Medicina Alternativa, associando recursos modernos como a acupuntura, ventosa terapia e termografia para tratamento pontual das lesões musculares? 
Tendo como intuito apresentar um relato de caso em que o objetivo é demonstrar que um recurso atual como a termografia pode complementar uma técnica milenar como a Acupuntura e a ventosa terapia.

\section{RELATO DE CASO}

Paciente, sexo masculino, 25 anos, oficial da Marinha do Brasil, militar de alto rendimento, procurou a acupuntura como terapia alternativa para auxiliar no tratamento de lesões musculares que estava sendo tratado por fisioterapeutas com o protocolo das imagens termográficas, apresentado excesso de suor, dor lombar, pulso profundo e rápido, língua sem saburra e seca e fortes dores musculares, tendo como queixa principal, lesão e fortes dores em membros inferiores.

Durante o tratamento, foram utilizadas agulhas de acupuntura $25 \times 30$ e ventosas de acrílico com procedimentos de ventosa terapia deslizante, a seco, e sangria. $O$ tratamento foi realizado uma vez por semana durante dois meses, alternando os acupontos conforme queixas do paciente, conforme imagens abaixo.

RC: 99476

Disponível em: https://www.nucleodoconhecimento.com.br/saude/ventosa-terapia 
Figura 1: Após treinamento intenso, paciente apresenta a primeira lesão, motivo pelo qual procurou o tratamento com a Medicina Alternativa.

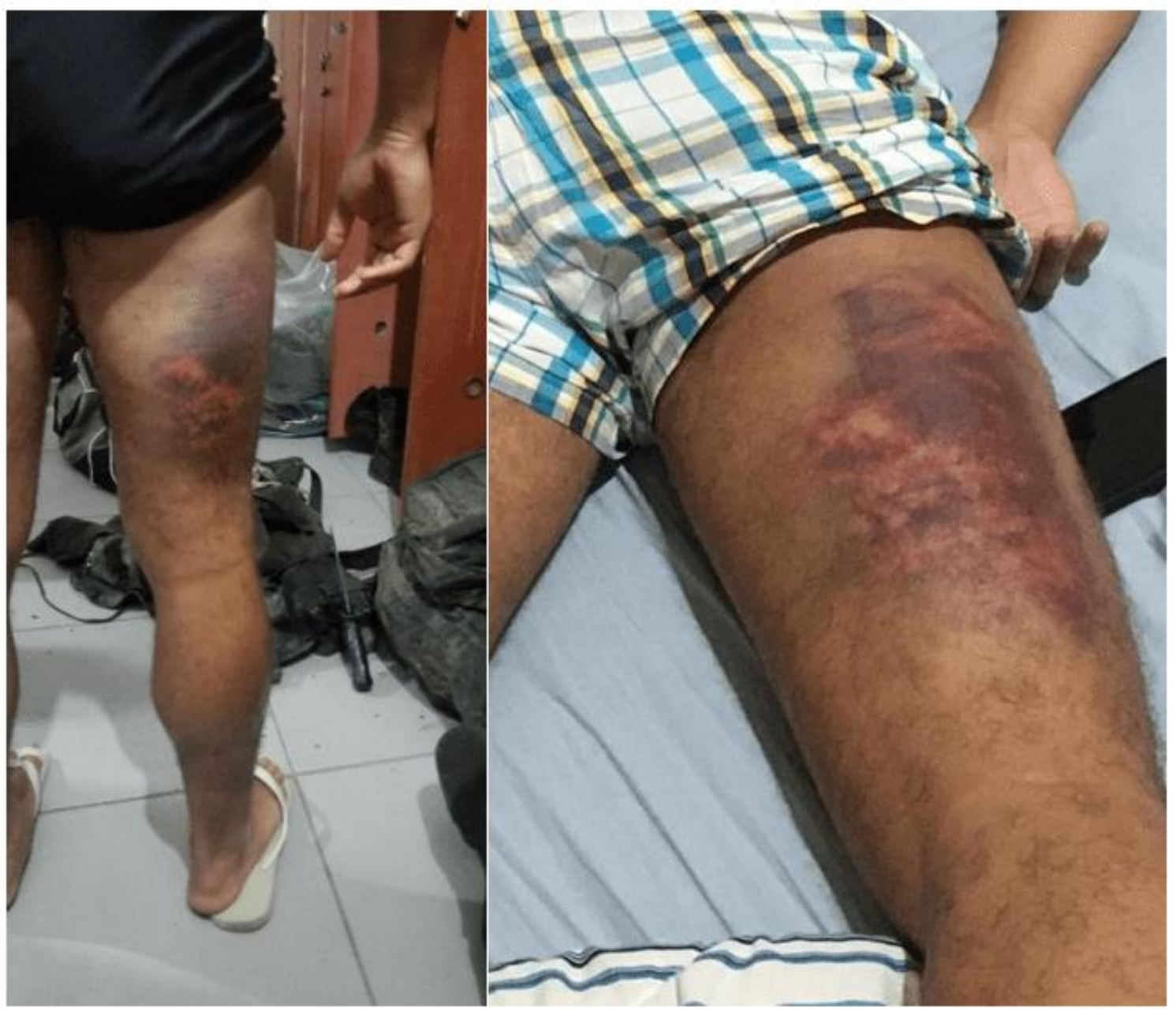

Fonte: Foto do próprio autor, 2019.

Na primeira fase do tratamento, foi realizado o diagnóstico energético da Medicina Tradicional Chinesa: Acometimento dos meridianos da Bexiga, Baço, Vesícula Biliar e Rim.

Primeiros pontos para equilibrar o paciente: IG4; BP6; IG11; F3.

Pontos usados durante o tratamento, conforme queixa principal do paciente: B40; B55; B56; B57; B58. Tais pontos foram determinantes para liberação dos meridianos acometidos. 
Imagem 2: Foto Termal mostra alta temperatura indicando lesão nas duas pernas apesar de visualização a olho nu da lesão somente na perna direita. Realizado tratamento bilateral com acupuntura.
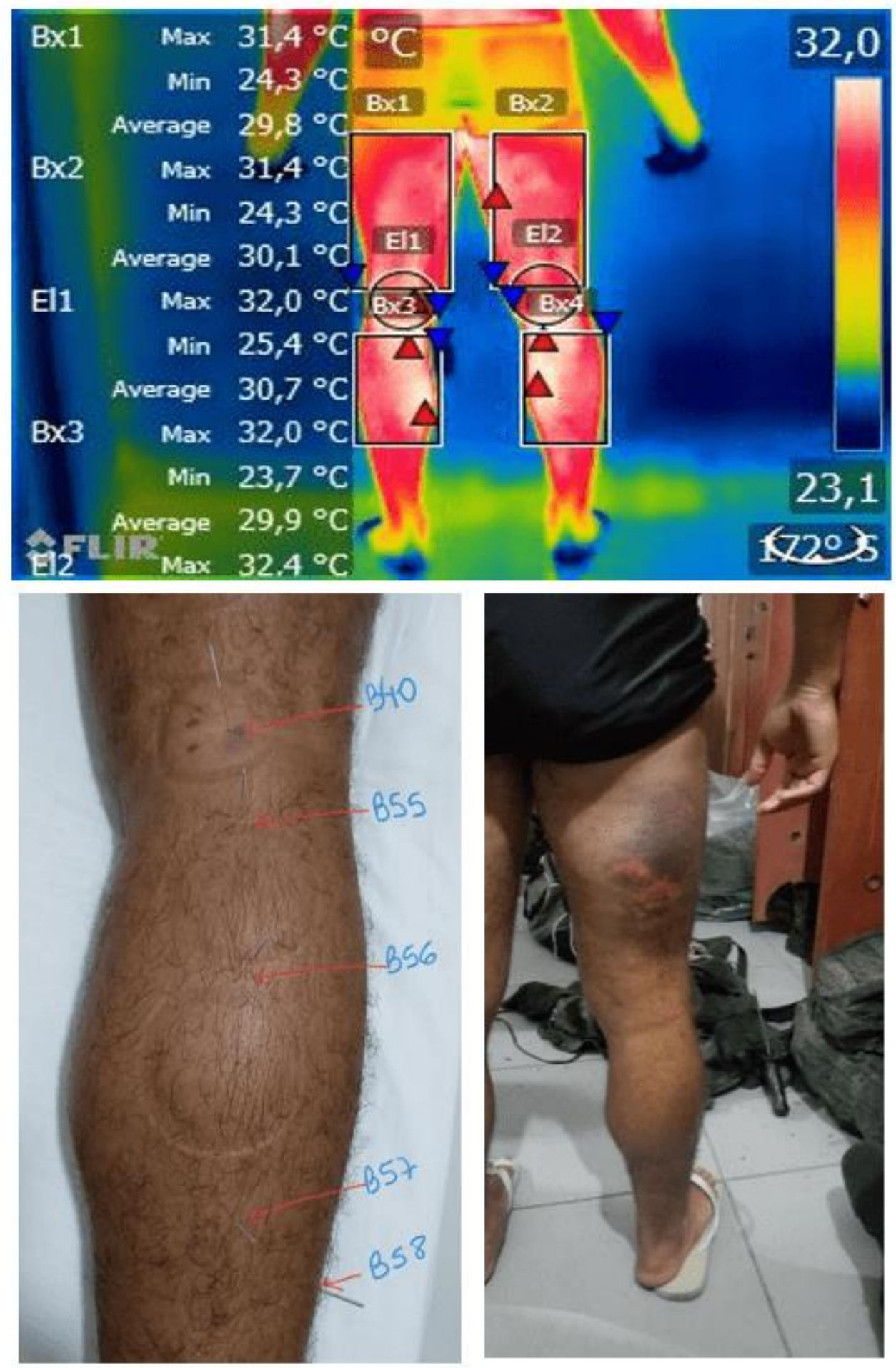

Fonte: Foto do próprio autor, 2019.

RC: 99476

Disponível em: https://www.nucleodoconhecimento.com.br/saude/ventosa-terapia 
Imagem 3: Tratamento com sessão de acupuntura uma vez ao dia. Resultado após 3 dias.

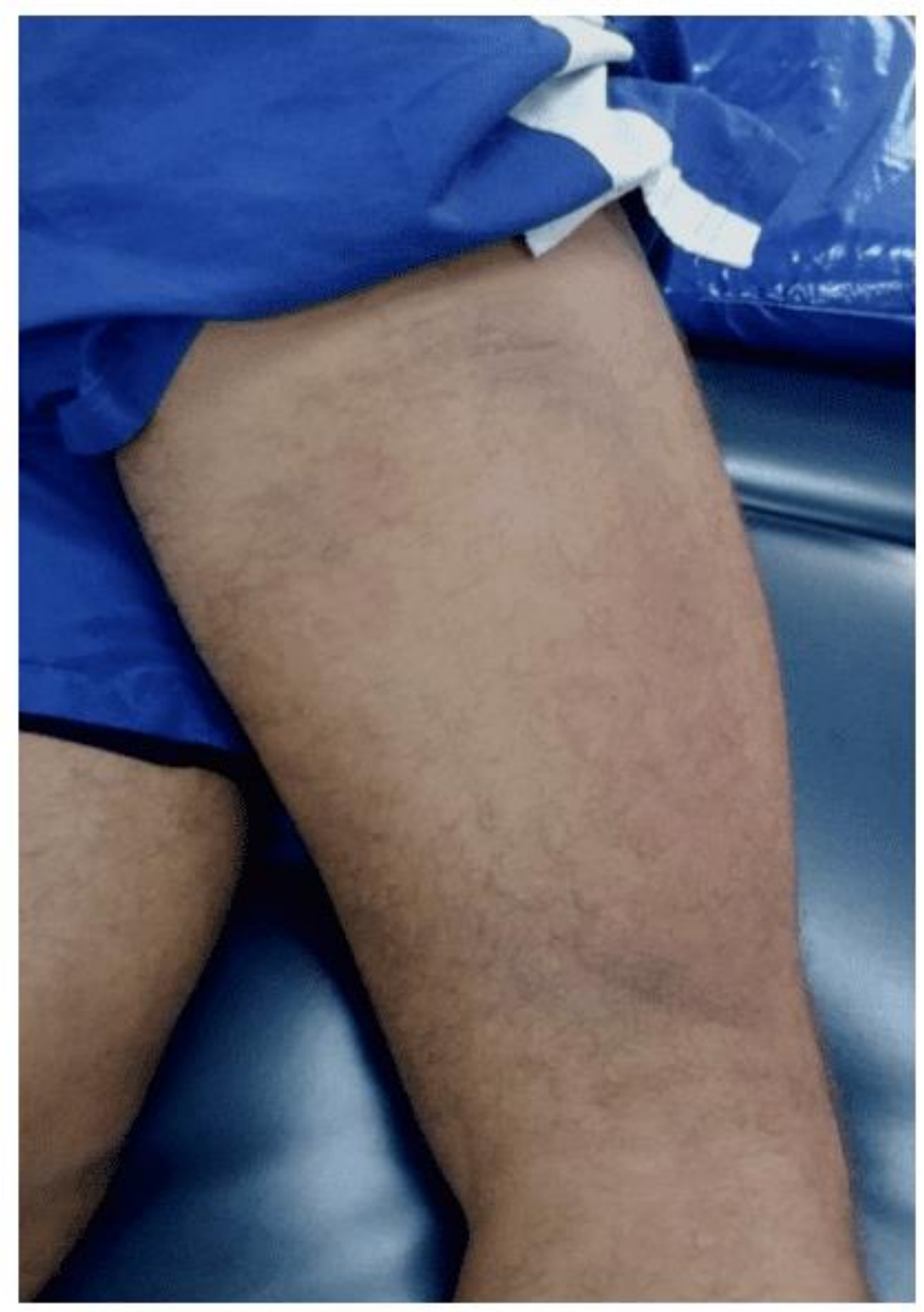

Fonte: Foto do próprio autor, 2019.

Nas semanas seguintes o paciente relatou fortes dores como demonstra a foto termal, por continuar realizando os treinamentos de alto impacto. 
Imagem 4: Foto termal, após queixa do paciente de fortes dores em membros inferiores, demonstrando mapeamento visual de distribuição da temperatura da pele.

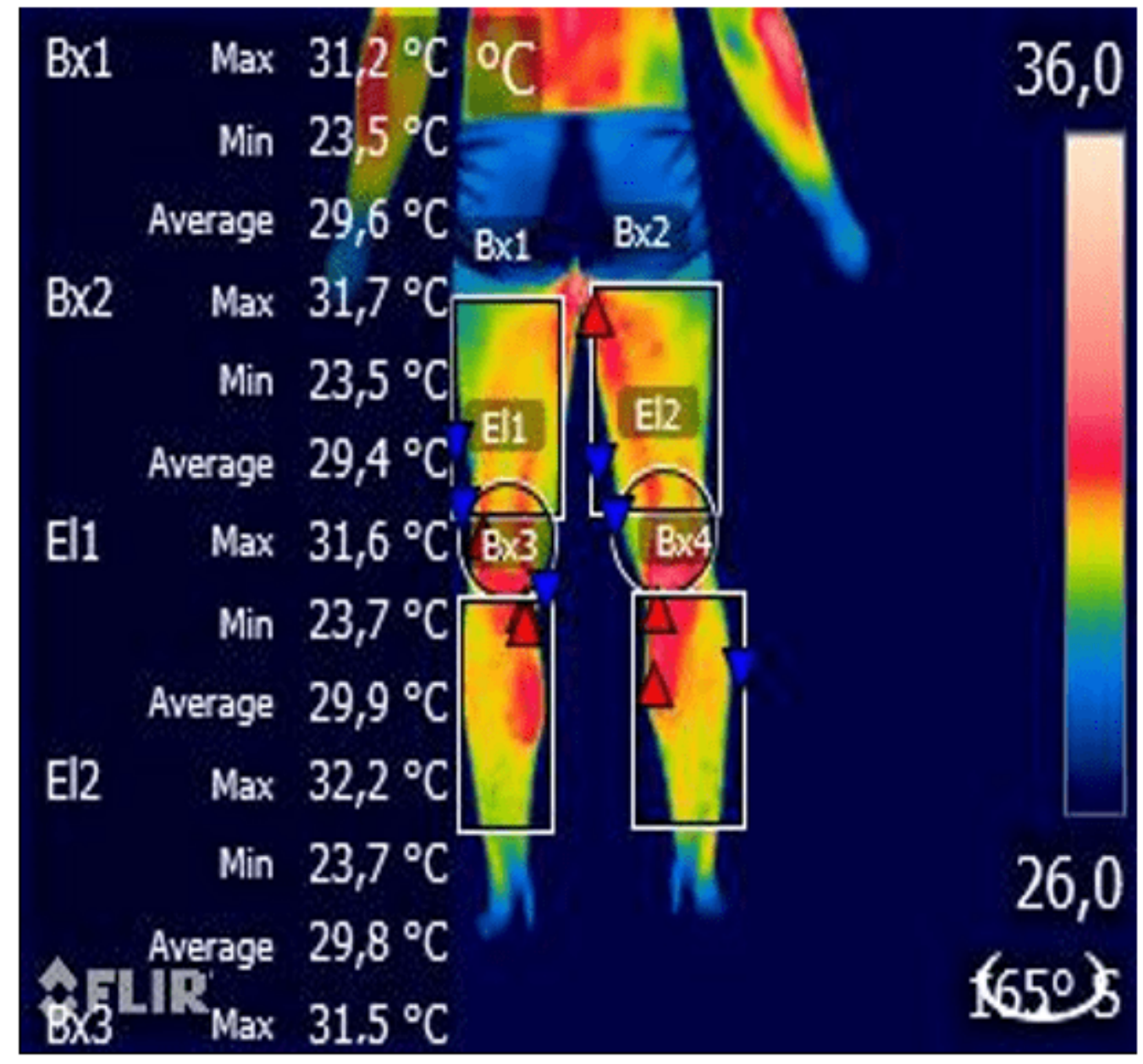

Fonte: Foto do próprio autor, 2019. 
Imagem 5: Fotos demonstrando aplicação de ventosa usando a técnica de sangria para liberar o fluxo nos meridianos acompanhando resultado das imagens termográficas.
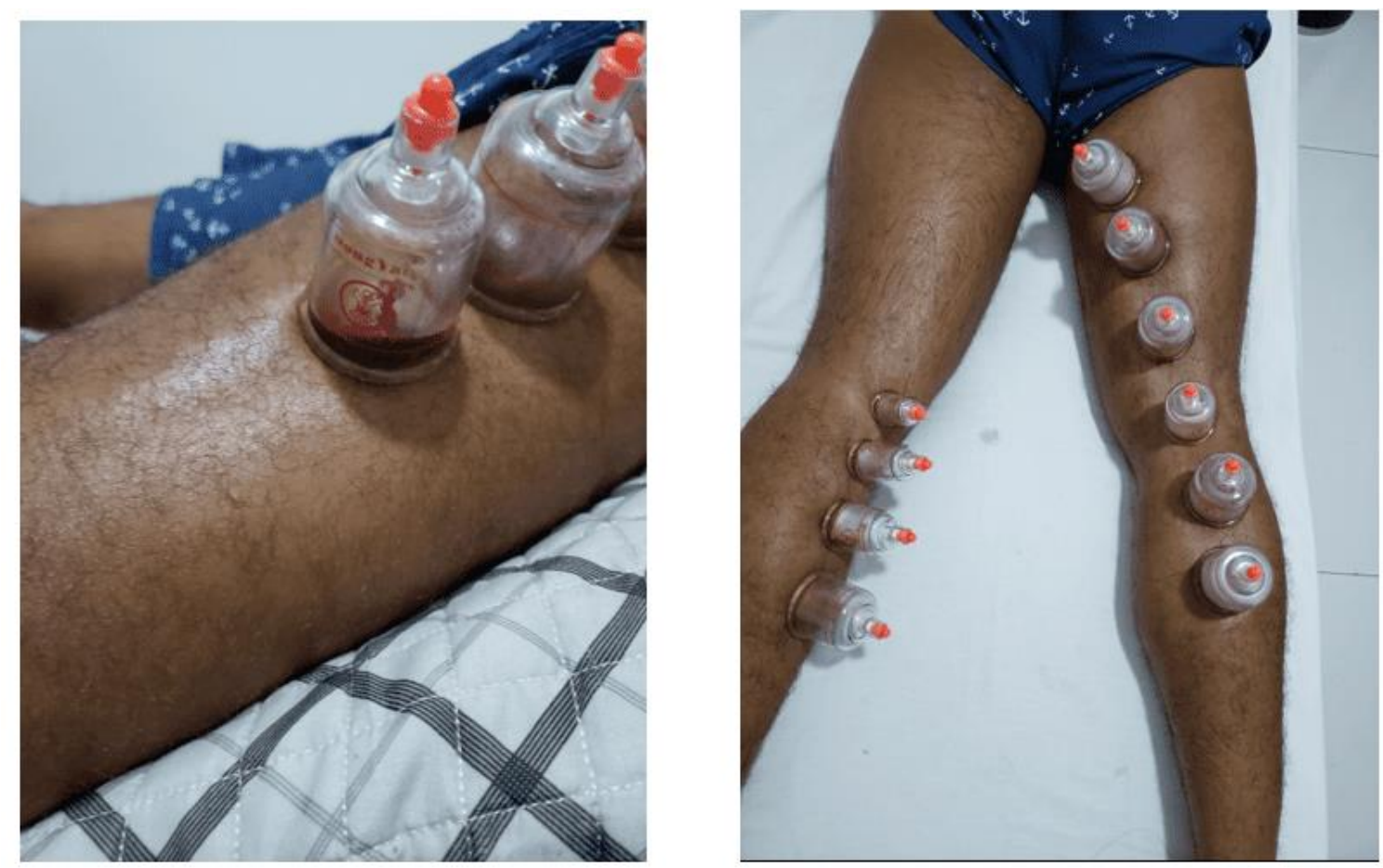

Fonte: Foto do próprio autor, 2019.

RC: 99476

Disponível em: https://www.nucleodoconhecimento.com.br/saude/ventosa-terapia 
Imagem 6: Foto termal realizada no final do tratamento, conforme fluxo sanguíneo e temperatura corporal, pode-se considerar foto de padrão normal.

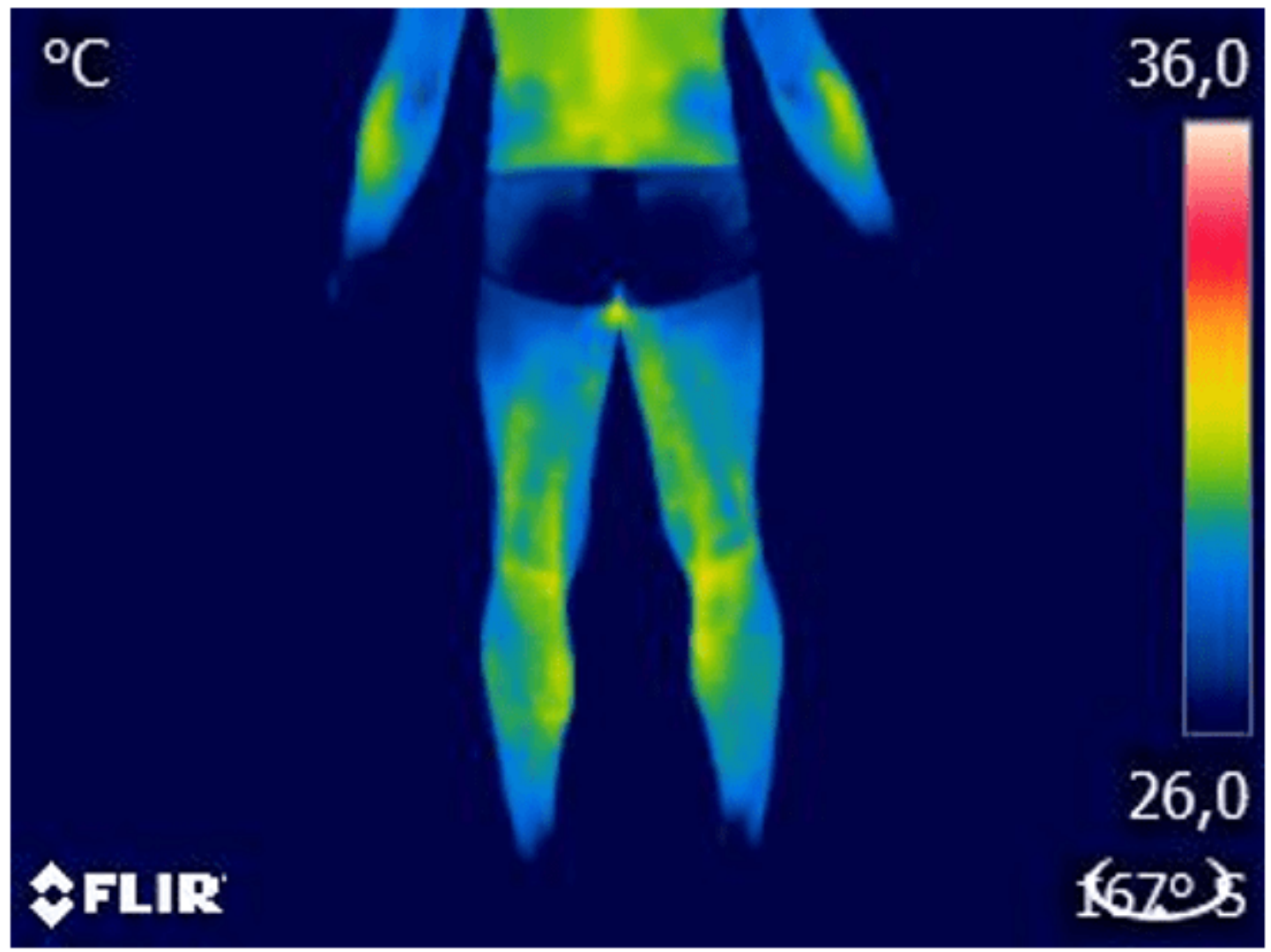

Fonte: Foto do próprio autor, 2019.

O paciente procurou um tratamento alternativo que foi acompanhado semanalmente pelo terapeuta acupunturista e pelo fisioterapeuta que realizava as fotos termais. Ambos os profissionais trabalharam em conjunto trocando informações para alcançar com rapidez e eficácia $100 \%$ de resultado no tratamento.

\section{DISCUSSÃO}

Todos os pontos de acupuntura utilizados durante a sessão foram escolhidos de acordo com o resultado da foto termal, tendo o paciente relatado melhora após as sessões. 
A termografia, de acordo com Brisochi (2005), "constitui-se em um exame revolucionário ao proporcionar a possibilidade de visualizar a sensação subjetiva da dor por exibição objetiva das mudanças na temperatura na superfície cutânea..."

Durante todo o tratamento foi utilizado ventosas seca, deslizante e com sangria, acupuntura sistêmica para estagnação e/ou estase nos meridianos, esfriar o sangue, tonificar o Qi e Xue, pontos Ashi com moxabustão.

As endorfinas desempenham um importante papel no funcionamento do sistema hormonal, além de ser muito mais potente que a morfina, daí a indicação e eficiência da acupuntura no tratamento de diversas dores. Além de relaxarem o corpo, as substâncias liberadas pela acupuntura também atuam na regulação de serotonina no cérebro. Alguns dos efeitos fisiológicos observados num tratamento contínuo com acupuntura são a redução de inflamações, o alívio da dor e espasmos musculares, além do aumento do número de células de defesa. (SIGNIFICADOS, 2011)

E como sugestão, o uso de spirulina diariamente, e com acompanhamento das fotos termais semanalmente.

No tratamento dos sintomas do paciente, foram utilizados os pontos de acupuntura abaixo relacionados, que apresentam (dentre outras) as seguintes funções de acordo com Maciocia (2018):

BP6 - Fortalece o Baço. Resolve umidade. Estimula a função do F. Suaviza o fluxo do Qi do F. Tonifica o R. Nutre o Xue e o Yin. Esfria o sague. Analgesia.

BP10 - Esfria o sangue. Revigora o sangue e elimina a estase. Nutre o sangue.

C6 - Revigora o sangue do C. Nutre o Yin do C. Interrompe sudorese. Acalma a mente.

CS6 - Move o Qi e o Xue. Revigora o Xue. Acalma a mente. Movimenta o Qi. 
E36 - Tonifica o Qi e Xue. Tonifica Qi original. Regula Qi nutritivo-Defensivo. Aumenta o Yang. Expele vento e umidade. Resolve edema.

F3 - Controla o Yang do Fígado. Suaviza o fluxo do Qi do F. Resolve umidade.

IG4 - Dispersa o Qi do Pulmão. Regula o Qi defensivo. Regula transpiração. Analgesia. Interrompe dor. Remove obstrução do canal. Tonifica o Qi. Harmoniza a subida e descida do Qi.

IG11 - Clareia o calor. Esfria o sangue. Remove obstruções. Remove calor no canal. R3 - Tonifica o Yin e o Yang do R. Acalma a mente. Beneficia a Essência. Fortalece a lombar e os joelhos

R6 - Nutre o Yin do R. Acalma a mente.

R7 - Tonifica o R. Abre a via das águas. Resolve edema. Fortalece a lombar. Regula sudorese

VC17 - Tonifica o Qi. Promove a descida do Qi.

VB34 - Remove obstrução no canal. Beneficia os tendões

VG20 - Controla Yang do F. Eleva o Yang. Eleva a mente e recupera a consciência.

B23 - Tonifica o Qi, Yang e Yin do R. Nutre a Essência do R. Fortalece a lombar. Nutre o sangue.

B26 - Fortalece a lombar

B40 - Clareia o calor. Esfria o sangue. Remove obstrução do canal.

B55 - Fortalece a região lombar.

B56 - Relaxa músculos e tendões das pernas 
B57 - Relaxa os tendões. Remove obstrução do canal.

B58 - Remove obstrução do canal. Fortalece o R.

Este são os Pontos Ashi conforme demonstrado por Maciocia (2018).

\section{CONCLUSÃO}

Embora as técnicas utilizadas possam ser usadas individualmente para o tratamento de lesões musculares, o estudo de caso, apresentado neste artigo, pode concluir que a Acupuntura com os recursos da Ventosa terapia e o auxílio da termografia para visualização pontual das áreas lesionadas, se mostraram eficientes para o alívio das dores musculares e para a eficácia do tratamento, tendo como alvo principal a melhora do paciente em seu bem-estar físico e mental, proporcionando ao paciente atleta de alto impacto o uso de técnicas sem a necessidade da utilização de remédios alopáticos. Após dois meses de tratamento, o paciente não apresentou lesões e já não se queixava de dor. Portanto, o caso apresentado possibilita ao terapeuta uma visão mais ampla para associar as várias opções de técnicas atuais para auxiliar no tratamento com a Medicina Tradicional Chinesa.

\section{REFERÊNCIAS}

BANDEIRA, F. et al. A termografia no apoio ao diagnóstico de lesão muscular no esporte. Revista Brasileira de Medicina do Esporte [online]. 2014, v. 20, n. 01, pp 59-64. Disponível em <htpps://doi.org/10.1590/S1517-86922014000100012>. ISSN 1806-9940. https://doi.org/10.1590/S1517-86922014000100012. Acesso em: 16 de nov. de 2019.

FILHO, Reginaldo. Ventosaterapia Chinesa. Editora Brasileira de Medicina Chinesa, 2016.

LIN, C.A. Da medicina tradicional chinesa à prática de acupuntura médica baseada em evidência. Revista de Medicina, [S.I], v.92, n.3, p.213-215, 2013. DOI: 10.11606/issn.1679-9836.v92i3p213-215. 
https://www.revistas.usp.br/revistadc/article/view/80001. Acesso em 05 de jun. de 2019.

MACIOCIA, Giovanni. Os Fundamentos da Medicina Chinesa. Terceira edição [Reimpr.]. Rio de Janeiro: Roca, 2018.

SOCIEDADE BRASILEIRA DE FISIOTERAPIA. O que é Termografia Clínica Funcional. 2014. Disponível em <htpps://www.sbf.org.br>blog>0 que-e-termo>. Acesso em: 29 de maio de 2019.

SIGNIFICADOS. Acupuntura. 2011. Disponível em https://www.significados.com.br/ Acesso em: 05 de jun.de 2019.

Enviado: Julho, 2021.

Aprovado: Outubro, 2021. 